
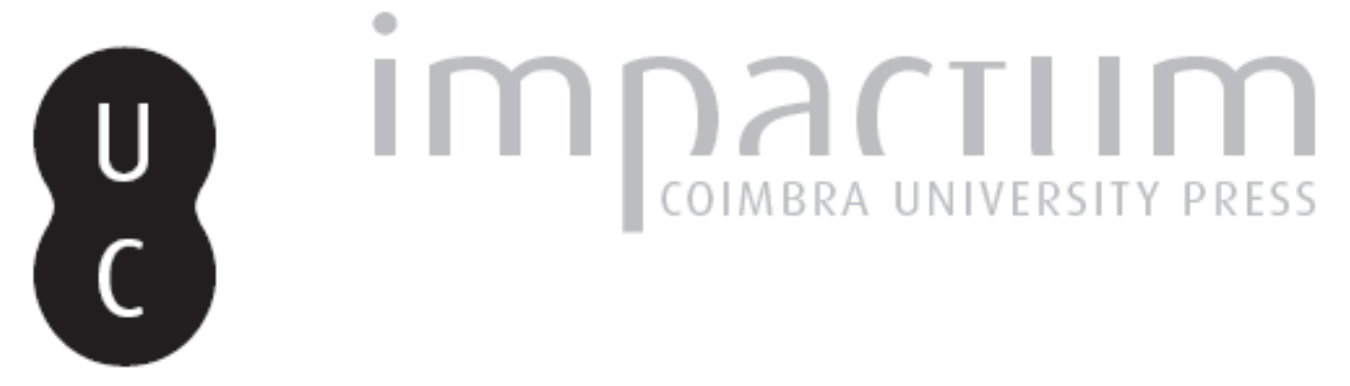

Una nueva (y sorprendente) inscripción de Termes (Hispania Citerior)

Autor(es): $\quad$ Gómez-Pantoja, Joaquín; Rodríguez Martín, F. Germán

Publicado por: Faculdade de Letras da Universidade de Coimbra

URL persistente:

URI:http://hdl.handle.net/10316.2/37706

DOI:

DOI:http://dx.doi.org/10.14195/1647-8657_43_8

Accessed : $\quad$ 26-Apr-2023 06:49:57

A navegação consulta e descarregamento dos títulos inseridos nas Bibliotecas Digitais UC Digitalis, UC Pombalina e UC Impactum, pressupõem a aceitação plena e sem reservas dos Termos e Condições de Uso destas Bibliotecas Digitais, disponíveis em https://digitalis.uc.pt/pt-pt/termos.

Conforme exposto nos referidos Termos e Condições de Uso, o descarregamento de títulos de acesso restrito requer uma licença válida de autorização devendo o utilizador aceder ao(s) documento(s) a partir de um endereço de IP da instituição detentora da supramencionada licença.

Ao utilizador é apenas permitido o descarregamento para uso pessoal, pelo que o emprego do(s) título(s) descarregado(s) para outro fim, designadamente comercial, carece de autorização do respetivo autor ou editor da obra.

Na medida em que todas as obras da UC Digitalis se encontram protegidas pelo Código do Direito de Autor e Direitos Conexos e demais legislação aplicável, toda a cópia, parcial ou total, deste documento, nos casos em que é legalmente admitida, deverá conter ou fazer-se acompanhar por este aviso.

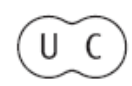


CONIMBRIGA

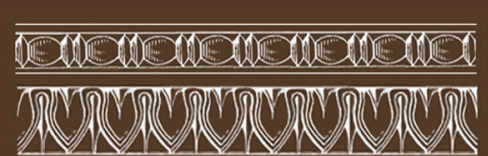

INSTITUTO DE ARQUEOLOGIA

VOLUME XLIII - 2004

FACULDADE DE LETRA

UNIVERSIDADE DE COIMBRA 
JoAQuín GÓMEZ-PANTOJA

Professor Titular da Universidad de Alcalá de Henares

(Departamento de Historia I y Filosofía)

F. ${ }^{\circ}$ Germán RodríGuez Martín

Archéologue. Ancien membre libre de la Casa de Velázquez

UNA NUEVA (Y SORPRENDENTE) INSCRIPCIÓN DE TERMES (HISPANIA CITERIOR)

"Conimbriga" XLIII (2004) p. 179-189

RESUMEN: Esta breve nota describe una inscripción latina descubierta en las excavaciones de Termes (Hispania Citerior) en 1997. Se trata de un fragmento de una gran lastra que contiene un documento legal y financiero, cuya característica más sobresaliente es la mención de cantidades milloniarias de sestercios.

SUMmARY: This short note deals with a new inscription found in Termes (Hispania Citerior) in 1997. The fragment, coming from a bigger block, contains a legal and financial document dealing with millions of sesterces. 
(Página deixada propositadamente em branco) 


\section{UNA NUEVA (Y SOPRENDENTE) INSCRIPCIÓN DE TERMES (HISPANIA CITERIOR)}

Al término de la campaña de excavaciones de 1997, José Luis Argente, director del Museo Numantino de Soria, me avisó del descubrimiento en Termes (Montejo de Tiermes, Soria), de dos nuevas inscripciones latinas: un bloque de piedra conteniendo lo que él calificaba como "documento económico", y los fragmentos de una placa de bronce, que me describió como una "tessera de hospitium". ${ }^{1}$ Dado lo avanzado de la estación, quedamos en la primavera siguiente para examinar ambas inscripciones en el Museo de Tiermes, donde habían sido depositadas. Pero cuando llegó ese momento, a Argente le habían diagnosticado ya un cáncer mortal y nunca más llegamos a vernos. Debido al interregnum que siguió a su muerte, no volví a tener acceso a esas inscripciones hasta que Elías Terés, actual director del Museo Numantino, me envió a principio de 2002 sendas fotografías de excelente calidad, que me permitieron leer y restituir ambas. El interés de la primera pieza - el "documento económico" - es tal que la presenté en la tercera jornada del Congreso de la Association Internationale d'Épigraphie Grecque et Latine, celebrado en Barcelona entre los días 3 a 8 de septiembre de 2002.2 El retraso en la publicación de las Actas del Congreso

1 El propio Argente publicó una brevisima noticia del hallazgo en Tiermes, Campaña de excavaciones 1977, Soria 1977: la primera pieza se describía someramente, sin foto ni lectura, mientras que de la segunda se daba una foto, que nos permitió ofrecer la transcripción provisional del texto en $H E p$. 7, 950. Otra mención al primer epígrafe, en J. L. GómEZ-PANTOJA, «Ex ultima Celtiberia. Desarrollo municipal y promoción urbana en las viejas ciudades arévacas», en C. CASTILlo et alii (eds.), Sociedad y economía en el Occidente romano, (Mundo Antiguo, Nueva Serie, 8), Eunsa, Pamplona, 2003, pp. 231-282.

2 Vid. la reseña de A. BLANCO en Boletín Informativo de la Sociedad de Estudios Latinos, 19, Diciembre 2002, pp. 15-18, esp. p. 16. 
y el ímpetu de los nuevos responsables del yacimiento de Tiermes han provocado que la editio princeps del epígrafe ya no sea la mia, sino la que acaba de aparecer en junio de 2004: sus autores ${ }^{3}$ la definen como una "primera y rápida aproximación al contenido del texto" y quizá sean esos rasgos los que han provocado algunos flagrantes errores de lectura y mis discrepancias con su interpretación del contenido y la finalidad del epígrafe. Ambas razones - y la amabilidad del editor de esta revista - me han inclinado a reproducir aquí lo más sustancial de la comunicación presentada en el Congreso de Barcelona, que sólo he modificado para enmendar o comentar la edición recién publicada y para incorporar algunas observaciones resultantes de un estudio más pormenorizado del epígrafe. ${ }^{4}$

Lo que nos ocupa es un bloque rectangular tallado en la caliza amarillenta local que fue profusamente empleada en la construcción de la ciudad. La regularidad de las dimensiones y la limpieza de la fractura por arriba y por el lado izquierdo demuestran que la pieza es el resultado del corte de una gran lastra inscrita, de la que sólo se conserva el costado derecho y el borde inferior. Las medidas máximas actuales son (62) x $(27,5)$ x $35 \mathrm{~cm}$, faltándole en la esquina superior izquierda una porción de forma casi cuadrada de $(18)$ x $(13,5)$ x $35 \mathrm{~cm}$. Ni el lateral derecho ni la trasera del bloque presentan rasgos dignos de reseñar y es probable que un defecto de la piedra o un corte inadecuado causase la fractura que atraviesa diagonalmente la pieza y la divide en las dos porciones desiguales en que nos ha llegado. Al quedar inutilizado para los fines a los que estuviera destinado, el bloque fue amortizado en los cimientos del muro meridional de una construcción - la taberna 22 aneja al llamado castellum aquae, junto al foro de la ciudad. ${ }^{5}$ La por-

3 J. MANGas y S. Martínez, "Nueva inscripción de Tiermes: propuestas para una discusión”, Gerión, 21-2, 2003, pp. 9-15, a partir de ahora citados edd.

4 Dedico esta breve nota en emocionado recuerdo de J. L. Argente: pero mi gratitud alcanza también a los vivos: E. Terés por haberme facilitado la autopsia de la inscripción y la excelente fotografía de A. Plaza que ilustra este artículo; E. Gutiérrez Dohijo, testigo del hallazgo de la inscripción, me aclaró algunos extremos del mismo; S. Martínez Carretero, actual responsable de Termes, discutió conmigo mi hipótesis y me adelantó algunos resultados recientes de las excavaciones; y J. P. Bost, L. Curchin, G. L. Gregori, P. Le Roux, J. Liz, E. Melchor, M. Navarro, J. M. Roddaz, M. Salinas, P. Sillières, A. U. Stylow e I. Velázquez son algunos de los colegas que gentilmente compartieron conmigo sus conocimientos y opiniones cuando leyeron este texto.

5 ARgente, cit. supra, pp. 32-33 y E. Gutíerrez Dohijo, per litteras, marzo 2002. 
ción que falta en la esquina superior izquierda modula las dimensiones actuales del bloque, lo que me hace sospechar que quizá falten otros 18 o $36 \mathrm{~cm}$ por arriba del primer renglón y un número indeterminado de múltiplos de $18 \mathrm{~cm}$ por el lado izquierdo. La anchura total de la inscripción se determinaría con fácilidad si pudiesemos reconstruir la datación consular de las dos última línea, lo que es imposible por ahora.

Del texto original resta la parte final de 17 renglones aunque, de ellos, uno terminaba antes de la parte conservada y del otro sólo queda un resto dudoso. Por lo ya dicho, supongo que faltan al menos dos o tres renglones por encima del primero conservado y un número indeterminado de letras por el lado izquierdo; salvo el desgaste de las letras junto al borde, el texto está completo por la derecha y puede suponerse, quizá, que hubiese un renglón corto por debajo del último ahora visible. Otros accidentes notables del epígrafe son la fractura ya aludida, que oblitera sendas letras de los rr. 7, 8 y 9, y el importante desconchón superficial del r. 6, que afecta diversamente a la integridad de cinco o seis caracteres. A primera vista, parece que el texto está distribuido en tres párrafos separados entre sí por líneas en blanco; sin embargo - y como ya he advertido - parece haber restos de una letra en el r. 3 y lo escrito en r. 13 obliga necesariamente a suponer su continuidad en la línea siguiente; del mismo modo, el numeral que cierra el r. 17 debía ir seguido de otro renglón, más corto que no alcanzaba la parte conservada. Las letras pueden describirse como capitales epigráficas de factura muy regular, aunque con algunos rasgos librescos. Su módulo va en disminución, de tal modo que una misma letra - la $\mathrm{A}-$, mide $4.35 \mathrm{~cm}$ en el r. 2; 3.95 en el r. 4; 3.06 en el r. 8 y sólo 2.6 en el r. 10. Es imposible determinar la altura de las letras del primer renglón, pero su anchura puede establecer una medida de comparación con las demás; así, la probable A del r. 1 mide 5,16 cm de ancha, mientras las citadas antes miden, respectivamente, 3,14; 2,25; 2,1 y 1,8 cm de anchura. Estas diferencias tipográficas permiten suponer, que los rr. 1-2 pertenecían a la inevitable praescriptio del documento, que iban seguidas por dos claúsulas separadas entre sí por un renglón corto.

Nótese el uso regular y sistemático de la diacrisis entre palabras; de la I longa para distinguir los numerales de las letras; del escaso recurso a la abreviación de palabras (r. 7: Termestinor(um)) y a los nexos (r. 9: [-] utabantur) y que la sigla del sestercio aparece en su forma prístina: dos fustes (el izquierdo siempre más alto), una $\mathrm{S}$ también sobrelevada y un rasgo horizontal que enlaza los tres elementos por sus medios. 
Lo más llamativo del epígrafe son, sin duda, sus notae numerales; la L (= quinquaginta), se escribe $\mathrm{G}$, mientras que la D (= quingenta), aparece sistemáticamente barrada (D); y, por último, el uso de los signos factorizantes: la línea sobre los numerales los multiplica por mil, mientras que, combinada con un paréntesis, multiplica por cien milb.

Mi lectura, con los suplementos y conjeturas que justifico a continuación, es la siguiente:

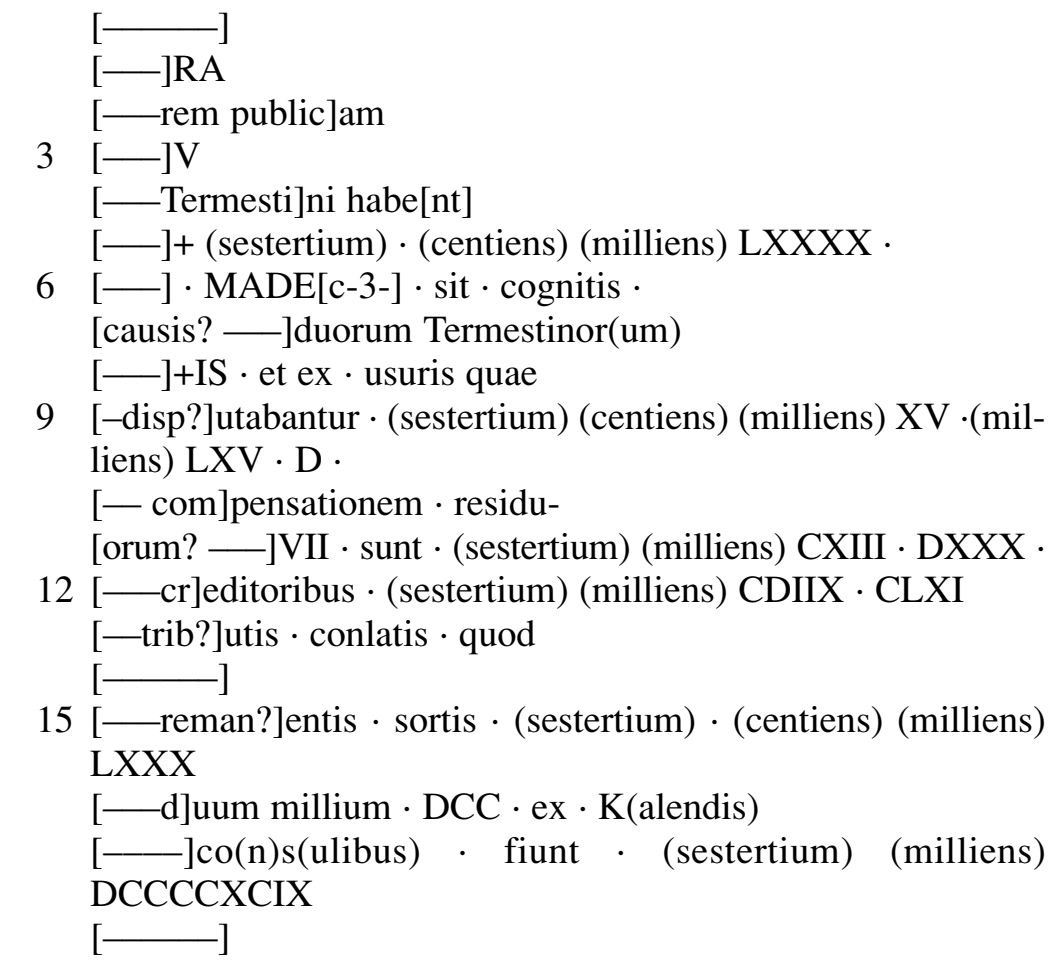

6 El procedimiento de expresar cantidades elevadas como resultado de su multiplicación por decies centena aut saepius, lo describe Plinio en $N H$ 33,47.113; ILS p. 798 , recoje una docena de ejemplos epígraficos, con cantidades que van desde el millón de sestercios hasta una cifra cercana a los 39.000.000. Vd. tambien, Th. Mommsen, GS, VII, p. 770; HuLtsch, RE 2 (1896), s.v. Arithmetica, cols. 1110-1114; y W. F. RichaRdSON, Numbering and Measuring in the Classical World, Auckland, 1985, pp. 4 y 6 . 
1: -$]++[-$, edd., pero se observan los rasgos inferiores de dos letras que, afortunadamente, son tan característicos que permiten su identificación. El mayor tamaño de las letras me inclinan a suponer que pertenecen a la praescriptio del documento - 2: la conjetura es muy incierta pero plausible si el renglón fue parte de la praescriptio - 3: omitida por los edd., quienes suponen un salto de párrafo, pero se aprecia en el mismo borde de fractura el ápice de una letra, probablemente $\mathrm{V}$ - 4: [-] NI HABE[1-2], edd.; lo conservado inclina a proponer un nombre masculino en nominativo plural que rige la forma verbal que sigue, posiblemente escrita con nexo, cf. infra r. 9; la restitución del nombre, aunque incierta, es adecuada al contexto - 5. De la primera letra visible sólo queda el ápice inferior de un asta vertical, quizá I; [-] HS LXXX (milia), edd., mal porque no han notado los dos trazos curvos que rodean al numeral y que multiplican la cantidad inclusa por 100.000, es decir, 9.000.000 de sestercios; la interpunción al comienzo y final del numeral - y dentro del cartouche factorizador -, indica que éste está completo - 6: MAD - SIC, edd., mal, porque antes de la rasura se observa el brazo inferior de otra letra; la palabra comienza en el mismo borde de rotura (es perfectamente visible la diacrisis previa) y no encuentro un suplemento adecuado, aunque si tengo algunas sugerencias que expondré en un próximo trabajo. Cognitis [causis] es una conjetura plausible - QUORUM, edd. La primera letra visible no puede ser ni una $S$ ni una Q (cf. rr. 8 y 14); por eso, sólo puede transcribirse como D o como $\mathrm{O}$ y dependiendo de la alternativa elegida (vid. infra), se debería leer [n]ovorum Termestinor(um) o duorum Termestinor(um) — 8: .TIS, edd. — 9: UTABANTUR, edd., entendiendo, al parecer, utor, mientras que mi propuesta apunta a computare o disputare; HS . XVI - LXVI, edd., i.e. 16.066 sestercios; pero un doble parentesis encierra XV y va seguido de diacrisis; se trata de otro "cartouche" factorizador similar al del r. 5, seguido de LXV sobrelineado, diacrisis y una D barrada. Los tres grupos de numerales van separados por interpunción, forzando al lector a operar mentalmente con las diversas cantidades: $((1000 \times 100 \times 15)+(1000 \times 65)+500)$, es decir, 1.565 .500 sestercios - 10: RESIDV, lapis, por lo que debe suponerse que la palabra, en genitivo, continuaba en el siguiente renglón - 11: Las tres primeras letras visibles son indudablemente un numeral, que pueden corresponder a una cantidad que, sumada con otra u otras (sunt), arroja el monto de 113.530 sestercios; pero también puede tratarse de una computo temporal, i.e. [—annis X]VII o similar - 12: [- CON]DITORIBUS, edd. 
Por delante de la primera letra visible queda el ápice superior de otra: editores parece impropio del contexto pero creditores cuadra bien con éste por ser un término habitual en el habla financiera y jurídica del latín; la cantidad que sigue (408.161 sestercios), se expresa distinguiendo con diacrisis los millares de las centenas; nótese el uso del arcaico IIX - 13: -]NTIS, edd., pero el primer rasgo es la parte superior de un brazo inclinado, que sólo puede corresponder a una $\mathrm{V}$; [trib] utis conlatis es, de nuevo, un suplemento plausible para el contexto - 14: omitida por los edd., pero el quod anterior obliga necesariamente la existencia de un renglón a seguido - 15: [PRAES]ENTIS SORTIS HS LXXX (milia), edd.; de nuevo, se pasa por alto la inclusión de la cantidad en un paréntesis que asegura que la cifra está completa (i.e. no es la repetición mutilada de la señalada en r. 5) y la multiplica por decies centena $=8.000 .000$ de sestercios; sors significa aquí caput, summa (i.e., oppositum usuris), por lo que puede suponerse que es el resto de los nueve millones mencionados: de ahí mi suplemento [reman]ens - 16: [-Tri?]UM MILLIUM DCC, edd.; pero justo en el borde de rotura queda el arranque del brazo derecho de otra $\mathrm{V}$, lo que permite reconstruir con seguridad el numeral; sorprende que la cantidad sea tan baja por comparación con las anteriores y que se exprese con números y palabras, pero es imposible determinar lo que había en la parte perdida de la inscripción; EX T[r?], edd.; claramente es el comienzo de una datación consular, cuyo final se conserva en el renglón siguiente - 17: DCCCCXCI, edd., entendiendo 991.000 sestercios, pero la línea sobre el numeral sobrepasa por la derecha la última cifra visible y cubría otra más que he comprobado que es una X; el resultado de este sumatorio final serían (fiunt) 999.000 sestercios.

La desgraciada mutilación del epígrafe hace que cualquier conjetura sobre su contenido, finalidad y circunstancias históricas deban discutirse con más detalle del que me permite el espacio concedido y mis propósitos iniciales; lo dejo, por lo tanto, para un próximo artículo y me concentro ahora en una serie de rasgos palmarios que merecen comentario por su singularidad. En primer lugar, es claro que la razón de ser del monumento es recordar una circunstancia o suceso en que los Termestini fueron agentes o pacientes directos. Además, la insólita presencia del adjetivo antes del etnónimo obliga a plantearse cuestiones inéditas, porque parece implicar una peculiaridad de la civitas de la que se carecía de noticia previa, salvo que se considere como sinoicismo el caso de los Dercinoassedenses, vicanii Cluniensium recordado en una 
tabla de bronce encontrada en los alrededores de la ciudad. ${ }^{7}$ Cualquiera de los dos suplementos del adjetivo (duo/novus) es plausible pero ninguno es obvio: en el estado actual de nuestros conocimientos, puede tratarse de un nuevo y desconocido cognomentum de la ciudad, a semejanza de los apellidos Novus/Vetus que califican a determinados topónimos hispanos; 8 o puede referirse a una fracción de sus habitantes, al estilo de otras de aparentes Doppelgemeinden hispanas. ${ }^{9}$

En segundo lugar, son dignas de consideración las cantidades de dinero en juego: en el r. 5, nueve millones de sestercios; en el r. 9, [disp?] utabantur 1.565 .500 sestercios; en el r. 11, sunt 113.530 sestercios; en el r. 13, ocho millones de [reman]entis sortis; y en los rr. 1617, ex k(alendis) [__ ] co(n)s(ulibus), 999.000 sestercios, sin contar los numerales incompletos de los rr. 11 y 16, que pueden ser sertercios $\mathrm{u}$ otra cosa. Probablemente no se trata de cantidades distintas, sino los resultados de diversos cálculos a partir de una cifra original (¿los nueve millones?) pero se encuentran en un rango de magnitud insólito en las inscripciones de otras provincias y son casi inéditos en las hispanas. ${ }^{10}$ Los millones de Termes nos devuelven al viejo problema del nivel de riqueza de las comarcas interiores de Hispania, sobre la que los datos disponibles arrojan visiones contrapuestas: Estrabón, por ejemplo, insistió machaconamente en la general escualidez de esas tierras, que forzaba a sus habitantes al pastoreo y el latrocinio; pero es precisamente él mismo quien también transmite el sorprendente dato (III, 4, 13) de que, en el 151 a. C., los celtíberos tributaron a Roma 600 talentos, es decir, casi 14 millones y medio de sestercios y otras fuentes confirman indirectamente la apreciación al afirmar repetidamente que la conquista de la zona pro-

7 A. d'Ors, «Un nuevo dato para la historia de la llamada Termancia», en Estudios dedicados a Menéndez Pidal, II, Madrid, 1951, pp. 21-30 = AE 1953, 267.

$8 \mathrm{Vd}$. los comentarios de A. U. StYlow. «Die Accitani veteres und die Kolonie Iulia Gemella Acci. Zum Problem von veteres, Alt-Stadt und Kolonie in der Hispania Ulterior.» Chiron 30 (2000), pp. 775-806 y M. PASTOR MUÑOZ, «Accitani veteres en una nueva inscripción latina de Piñar (Granada)», HAnt, 24,(2000), pp. 155-164, al respecto de la nueva inscripción de Piñar (Granada).

9 Vd. los Valentini veteres et veterani (CIL II²/14, 14-19; 21-22; y 24-26 o el ordo Singilensium vetus (CIL II²/5, 792 y 794), sin olvidar la cita de Estrabón (III, 2,15) sobre la extracción híbrida de los habitantes de Emerita Augusta, Caesarugusta y Pax Augusta,

10 El único caso hispano equiparable es la famosa benefacción de Q. Torius Q.f. Culleo en Castulo, vd. CIL II, 3270 = AE 1975, 526. 
porcionó a los romanos botines dignos de mención. Accesoriamente, la notación numeral suple en parte la perdida de la fecha que comienza al final del r. 16 y terminaba con el preceptivo co(n)s(ulibus) en el siguiente renglón. El empleo de G para notar quinquaginta era una práctica corriente en Roma y sus alrededores con Augusto pero demodé después aunque, en Hispania, el uso parece haber resistido hasta època tiberiana; 11 esta fecha coincide con los rasgos paleográficos de la inscripción - el tipo de letras, las cifras sustractivas (IIX por VIII) - y con el contexto arqueológico del hallazgo, calificado como "altoimperial" por Argente, lo que parece significar "flavio" o anterior. 12 Estas características contradicen rotundamente la datación en el s. II d. C. asignada por los edd. y forzada, en mi opinión, por el prejuicio de considerar que se trata de los estatutos de una fundación alimenticia. ${ }^{13}$

$\mathrm{Y}$, finalmente, es destacable el léxico empleado, que connota tal familiaridad con las instituciones legales y financieras romanas que puede resultar fuera de lugar en una inscripción temprana y procedente de un oppidum de la profunda Celtiberia; ${ }^{14}$ pero los últimos veinte años nos han acostumbrado a la facilidad con la que un nuevo hallazgo es capaz de trastornar la communis opinio en esta y otras materias. Ya se ha dicho que la mutilación hace difícil precisar el contenido y propósitos del texto, pero las palabras no ocultan el ambiente en que se gestó el documento. El uso de compensatio, creditores, sortes y usura apunta a la terminología técnica y legal de argentari, feneratores y mensari, mientras que cognitis [causis] y [disp] utabantur señalan que puede tratarse de un litigio resuelto por un magistrado; y si ese es el caso, la mención de la [com]pensatio residu[orum] de los rr. 10 y 11 y de [trib]uta conlata en r. 13, puede indicar la naturaleza de la actio in litis: un pleito sobre el mal uso del dinero público (recuérdese la lex Iulia de peculatu,

11 J. S. Gordon y A. E. Gordon, Contributions to Palaeography of Latin inscriptions, Berkeley, 1957 (reprint, Milano 1977), p. 181; los ejemplos hispanos son CIL II, 4701 y 4703; y 4712, 4715, 4717 y 6208; todos ellos son miliarios encontrados en los alrededores de la Col. Patricia, los dos primeros del 2 a. C. y los otros cuatros de los años 35-39 d. C.

12 ARgEnTE, cit. supra, p. 14.

13 Mangas-Martínez, cit. supra. p. 12.

14 Vd. L. A. Curchin, «Juridical Epigraphy and Provincial Administration in Central Spain», en J. GonZÁLEZ (ed.), Roma y las provincias. Realidad administrativa e ideología imperial, Madrid, 1994, pp. 87-102. 
sacrilegiis et residuis) ${ }^{15}$ o sobre deudas tributarias en la que los Termestini o una porción de ellos, fueron los acredores (r. 12: [cr]editores) o, más probablemente, los deudores. $\mathrm{Y}$ en tal escenario, resulta pertinente lo que Tácito dice sobre Termes en un famoso pasaje frecuentemente citado por motivos distintos al del verdadero punto interés del relato; me refiero, claro está, a Ann. 4, 45, donde se narra la emboscada que en el 25 d.C., un termestino tendió al praetor provinciae L. Pisón, causándole la muerte; los comentarios se han fijado sobre todo en que el criminal no dominaba el latín, pero la cuestión importante para Tácito y sus contemporáneo era, sin duda, el asesinato de un magistrado imperial cuando trataba de llevar a cabo su provincia, que no era otra que recuperar pecunias e publico interceptas, indudablemente por los termestinos, que no dudaron en recurrir a la conjura homicida para evitarlo.

Es posible, pues, que la combinación del nuevo hallazgo de Termes con el pasaje de Tácito sirvan para iluminar un oscuro incidente del reinado de Tiberio, pero antes de dar por segura esa relación debe asegurarse con mayor precisión la interpretación del epígrafe, su cronología y el contexto arqueológico en que apareció. Eso pretendo hacer en un próximo trabajo. En cualquier caso, me parece evidente que el descubrimiento póstumo de José Luis Argente cambia por completo nuestra percepción de Termes e incita a seguir buscando en ese yacimiento las claves de su pasado.

15 Paul. Sent. 5, 25 (Krüger); Dig. 48.13; Cod. Iust. 9, 28. 


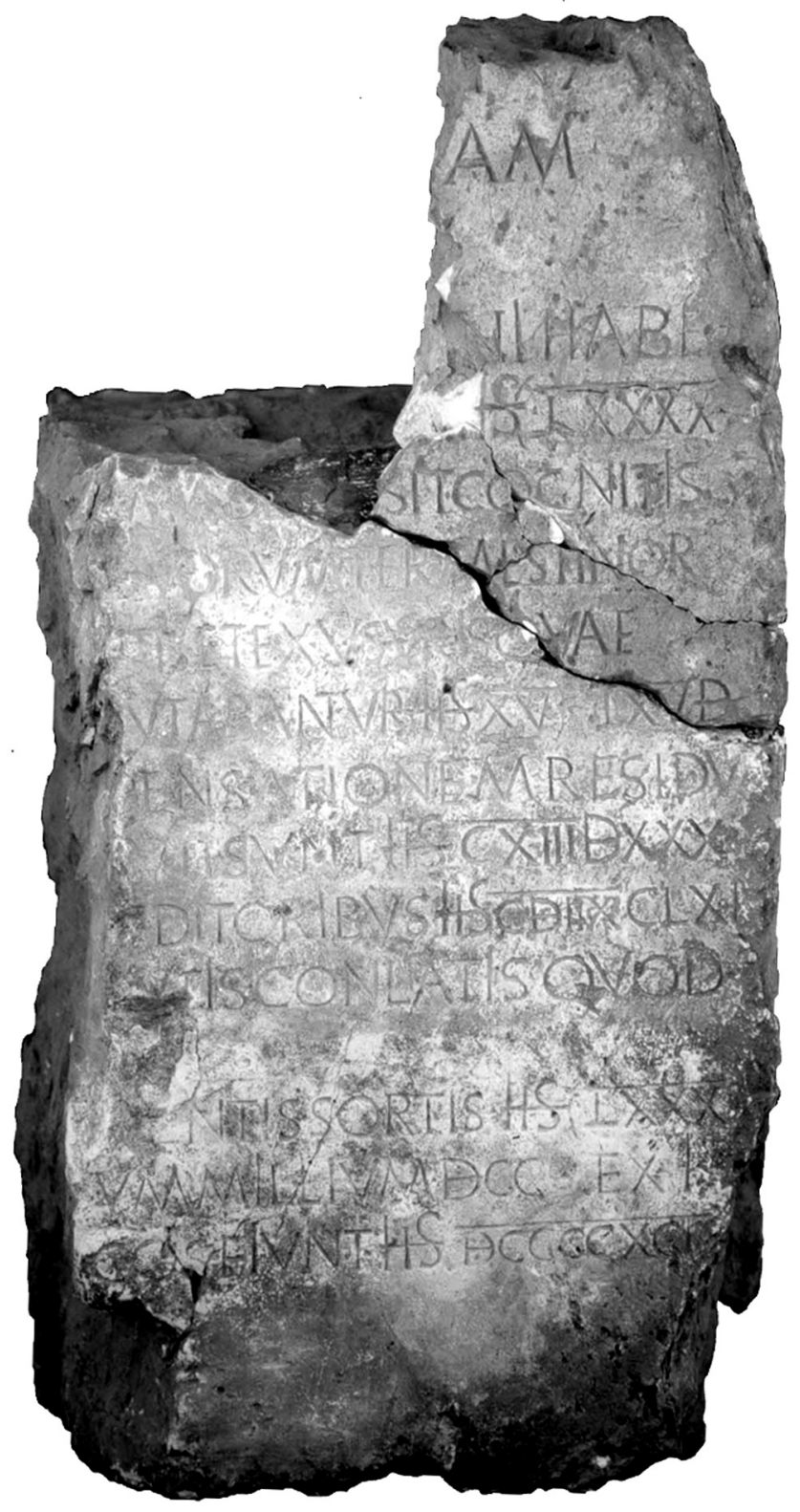

